\title{
Berta Katscher
}

\section{Einblicke in ihr Leben und Schreiben}

Susanne Blumesberger

\section{Die fast Vergessene SCHRIfTStellerin, ÜBERSETZERIN UND JOURNALISTIN}

Die Erzählerin, Übersetzerin und Feuilletonistin Berta Katscher (1860-1903), die auch unter den Pseudonymen Ludwig Ungar, Albert Kell(n)er, Ludwig Koelle, Bela Keleti und Ludmilla Koelli veröffentlichte, die sie sich mit ihrem Mann teilte, verbrachte ihre Jugend in Ungarn, der Türkei und in der Hercegovina. Nach der Heirat mit dem Schriftsteller Leopold Katscher lebte sie in London, Berlin, Baden bei Wien und Wien, schließlich ab 1897 in Budapest, wo sie am 16. September 1903 starb. ${ }^{1}$ Sie verfasste zunächst journalistische Texte, die u.a. in der Frankfurter Zeitung erschienen; nach einigen Reiseberichten begann sie sich v.a. gesellschaftspolitisch $\mathrm{zu}$ engagieren, trat für den Kinderschutz, den Tierschutz und für die Friedensidee ein und arbeitete u.a. an der monatlich erscheinenden Zeitschrift der österreichischen Friedensgesellschaft Die Waffen nieder!, herausgegeben von Bertha von Suttner, mit. Ihre Friedensbestrebungen flossen auch in ihre eigenen Werke ein. Nebenbei übersetzte sie aus dem Englischen und Ungarischen und verfasste Buchkritiken. Ihre Arbeiten geben einen tiefen Einblick in das gesellschaftliche Leben Österreichs um die Jahrhundertwende. Auch ihre Tochter Rosika Schwimmer (1877-1948) war Pazifistin und setzte sich für die Rechte von Frauen ein.

Ihr Nachlass befindet sich in der New York Public Library, Humanities and Social Sciences Library Manuscripts and Archives Division. ${ }^{2}$ Unter den deutschsprachigen Schriftstellerinnen aus dem Gebiet der heutigen Slovakei zählt sie heute zu jenen, deren Wirken noch relativ unerforscht ist. ${ }^{3}$ Dieser Beitrag soll Berta Katscher, die in mehreren Ländern und Kulturen lebte, wieder in Erinnerung rufen.

1 | Zur Biografie s. auch Blumesberger, Susanne: Handbuch der österreichischen Kinderund Jugendbuchautorinnen. Bd. 1: A-L. Wien: Böhlau 2014, S. 555-557. Siehe auch den Beitrag von Ingrid Puchalová im vorliegenden Band.

2| Leopold and Berta Katscher Papers 1866-1939. MssCol 6318.

3 | Vgl. Meier, Jörg: Deutschsprachige Schriftstellerinnen des 18.-20. Jahrhunderts aus dem Gebiet der heutigen Slowakei. In: Hörner, Petra (Hg.): Vergessene Literatur - Ungenann- 


\section{Berta Katschers Aufstieg zUR VIELBEACHTETEN SCHRIFTSTELLERIN}

Berta Katscher, am 12. Juni 1860 in Trenčín/Trencsén/Trentschin geboren, stammt aus einer kinderreichen jüdischen Familie, ihr Vater war Joseph Katscher, ihre Mutter Therese, geborene Blumgrund. Als sie zwei Jahre alt war, starb ihre Mutter. Ihre älteste Schwester übernahm die Erziehung und ging dabei sehr streng mit dem Kind um. Märchen bedeuteten dem kleinen Mädchen zu dieser Zeit sehr viel. Es erfand selbst welche und erzählte sie den Freundinnen. Später kam Berta Katscher zu Pflegeeltern und musste ihnen, als sie 13 Jahre alt war, in die Hercegovina folgen. Dort war sie zumeist im Haushalt beschäftigt. Das änderte sich jedoch nach ihrer Eheschließung, denn ihr Mann, ihr Cousin Leopold Katscher (1853-1939), den sie am 17. Mai 1881 in der Synagoge in Temesvár/Timișoara heiratete, war stolz darauf, eine Schriftstellerin zur Frau haben.

Leopold Katscher studierte Medizin, Rechtswissenschaften, Volkswirtschaft und Literaturgeschichte an den Universitäten Wien, Budapest und London und setzte sich in seinen Werken v.a. mit pazifistischen und sozialreformatorischen Ideen auseinander. Er griff in seinen Texten Themen wie das Frauenstimmrecht, die Gefängnisreform und die Abstinenzbewegung auf. Leopold Katscher, der zusätzlich zu jenen Pseudonymen, die er gemeinsam mit seiner Frau benutzte, auch unter Kosmopolit, Heinanus, Hermann, Qudam, Spectator und Anglicus schrieb, gab mehrere Zeitschriften sowie die Buchreihe Österreichisch-Ungarische Volksbücher heraus. Er war auch (Mit-)Begründer mehrerer Vereine, wie 1895 der Ungarischen Friedensgesellschaft und 1896 des Fiumaner Friedensvereins. Ab 1891 war er Vorstandsmitglied der Österreichischen Friedensgesellschaft. Außerdem war er Vorsitzender der Europäischen Kommission für Sozialreform und Mitbegründer des Deutschen Schriftstellerverbandes. Für sein Engagement erhielt er die Goldmedaille der Pariser Weltausstellung und die Silbermedaille der Charles-RobertStiftung. Dieses Umfeld eröffnete natürlich auch seiner Ehefrau Berta Katscher zahlreiche Möglichkeiten. Sie engagierte sich ebenfalls in den genannten Bereichen und arbeitete an mehreren seiner Werke mit. Als Beispiel sei hier nur sein Buch Schuldlos verurteilt! ${ }^{4}$ genannt. An vielen Publikationen wirkte das Ehepaar gemeinsam mit, u.a. an der zum Teil in drei Sprachen (Ungarisch, Deutsch, Französisch) publizierten Halbmonatsschrift Az Ezeréves Magyarország (Das tausendjährige Ungarn, 1896-1898). Berta Katscher ist darin u.a. mit der Humoreske A hajtóvadászat (Die Treibjagd) vertreten. ${ }^{5}$ Ihre ersten beiden Arbeiten erschienen in der Frankfurter Zeitung und der Wiener Zeitschrift Die Heimat. 1886 publizierte sie in der Deutschen Wochenschrift. Organ für die gemeinsamen nationalen Interessen Öster-

te Themen deutscher Schriftstellerinnen. Frankfurt a.M. u.a.: Peter Lang 2001, S. 241-262, hier S. 241.

4 | Katscher, Leopold: Schuldlos verurteilt! Anregungen, Betrachtungen, Erzählungen. Leipzig: Janssen 1895. Vgl. Suttner, A[rthur] G[undaccar] v.: Schuldlos verurteilt. In: Wiener Montagspost v. 12.11.1894, S. 5.

5 | Kácser, Berta: A hajtóvadászat [Die Treibjagd]. In: Az Ezeréves Magyarország 2 (1897), S. 21. Vgl. die Annonce in Pester Lloyd v. 2.5.1897, S. 8. 
reichs und Deutschland den Beitrag Verheirathet - dennoch glücklich.$^{6}$ Im selben Jahr schrieb sie das Feuilleton Klagebrief einer Strohwitwe, in dem sie sich auf humorvolle Weise über die Tatsache beklagt, dass das Ausgehen von Strohwitwern ohne Begleitung als selbstverständlich akzeptiert wird, sich alleine vergnügende Strohwitwen jedoch eher kritisch betrachtet werden. ${ }^{7}$ Sie unternahm zu dieser Zeit viele Reisen und hielt sich zeitweise auch in London auf. Dies regte sie zu ethnografischen Skizzen an, die sie u.a. in der Wiener Mode (1887-1938) veröffentlichte, die sich Modethemen, Kochrezepten, Theater- und Filmberichten widmete und auch Kurzgeschichten publizierte, weiters in der Münchner Allgemeinen, in der Leipziger Illustrirten (1843-1944), für die u.a. auch Friedrich Hebbel, Theodor Storm oder Karl May schrieben, in der Leipziger Zeitung und im Bazar. Später setzte sie sich für den Tierschutz und für die Friedensidee ein, schrieb gegen Justizirrtümer und gegen Spiel- und Trunksucht. Berta Katscher wirkte auch an der Zeitschrift Die Waffen nieder! (1892-1899) von Bertha von Suttner mit. Am 10. Mai 1895 hielt sie im Rahmen des Suttner-Abends, veranstaltet von der Österreichischen Gesellschaft für Friedensfreunde, den Vortrag Die geraubte Uhr. ${ }^{8}$ Sie beteiligte sich auch an der Anthologie Wiener Liebesgaben. Zum Besten der Wiener freiwilligen Rettungs-Gesellschaft (1892), in der auch Beiträge zahlreicher anderer Autorinnen und Autoren erschienen, unter ihnen auch von Marie von Ebner-Eschenbach (1830-1916), der Vertreterin der realistischen österreichischen Heimaterzählung, Aphoristikerin und Verfasserin autobiografischer Schriften. ${ }^{9}$ Nebenbei war Berta Katscher für bedeutende Zeitschriften als Übersetzerin aus dem Englischen und Ungarischen tätig und verfasste für mehrere literarische Blätter Buchkritiken. Zahlreiche Novellen, Humoresken, Feuilletons und Kritiken erschienen unter ihren Pseudonymen. Insgesamt arbeitete sie im Zeitraum 1882 bis 1894 an mehr als 76 Zeitschriften mit, verfasste in dieser Zeit neben drei eigenen Büchern und sechs Übersetzungen 121 Beiträge. ${ }^{10}$

\section{Kampf für den Frieden und gegen die Armut}

Berta Katscher setzte sich in ihren Werken - meist zusammen mit ihrem Mann sehr oft mit gesellschaftspolitischen Fragen auseinander. Sie griff Probleme wie Trunksucht, unverschuldete Armut ebenso auf wie die weit verbreiteten Vorurteile gegen die als »Blaustrümpfe« bezeichneten Frauen, die statt einer Versorgungsehe Bildung, Beruf und Selbstständigkeit anstrebten. In ihren Werken, von denen ei-

6 | Katscher, Bertha: Verheirathet, aber dennoch glücklich! In: Deutsche Wochenschrift. Organ für die gemeinsamen nationalen Interessen Österreichs und Deutschlands v. 28. 11.1886, S. 611-612. Vgl. die Annonce in Salzburger Volksblatt v. 3.12.1886 [S. 5].

7 | Katscher, Berta: Klagebrief einer Strohwitwe. In: Prager Tagblatt v. 24.7.1886, S. 1-2.

8 | Vgl. NN: Die Österreichische Gesellschaft der Friedensfreunde. In: Neue Freie Presse v. 13.4.1895, S. 6.

9 | Vgl. Katscher, Berta: Die beiden Todten. Bluette in einem Aufzug. In: Wiener Liebesgaben. Zum Besten der Wiener freiwilligen Rettungs-Gesellschaft. Wien/Leipzig: Max Merlin 1892, S. 97-114.

10 | Hacker, Lucia: Schreibende Frauen um 1900. Rollen - Bilder - Gesten. Berlin u.a.: LIT 2007, S. 123. 
nige erst postum veröffentlicht wurden, gibt sie auch Hinweise, wie sich junge Menschen richtig zu verhalten haben, und zeigt Humor, wie beispielsweise in der Geschichte Der versteigerte Rock, die 1890 in der Anthologiereihe Österreichisch-Ungarische Volksbücher erschien. ${ }^{11}$ Bei dieser kurzen Geschichte, die von zwei Freunden handelt, der eine eitel und gut aussehend, der andere eher nachlässig, steht das Vertauschen und schließlich die Versteigerung eines Salonrockes, den zufällig der künftige Schwiegervater des einen ersteigert, im Vordergrund.

Im selben Jahr veröffentlichte sie unter dem Titel Weihnachtsgeschichten ${ }^{12}$ fünf Erzählungen, Weiße Rose, Schani, Was dann?, Fritzchens Weihnachten und Die alte Uhr. In der Geschichte Weiße Rose erkennt ein junges Mädchen erst auf den zweiten Blick, dass sie einen jungen Mann, der schon länger um sie wirbt, liebt. Sie weist ihn immer wieder ab, erst als er zu einem Trick greift und den unansehnlichen Schuster zu Weihnachten anstiftet, auf der Kirchenschwelle auf sie zu warten, was einem Aberglauben nach den Bräutigam anzeigen soll, erkennt sie die Liebe zu ihm. Schani, die zweite Geschichte, handelt vom Sohn eines armen Flickschusters, der zu Weihnachten Stiefel austragen soll, um für die Familie Geld für das Weihnachtsessen zu verdienen. Er gerät jedoch auf der Straße mit einem Jungen in Streit, verliert die Stiefel und traut sich nicht mehr heim. Aus Hunger stiehlt er der Marktfrau einen Apfel, wird erwischt und beinahe zur Polizei gebracht. Eine reiche Frau hat Mitleid mit ihm, möchte ihn sogar adoptieren, beschenkt jedoch schlussendlich die Familie des Jungen reichlich und sorgt dafür, dass später sein sehnlichster Wunsch in Erfüllung geht und er studieren kann. In Was dann? kommt ein verarmter Schriftsteller zufällig in das Haus eines Wohltäters, den einst sein eigener Großvater gerettet hat. Er bekommt von ihm nicht nur Unterstützung, sondern schließlich auch die Hand seiner Tochter. Weniger positiv endet die Geschichte Fritzchens Weihnachten: Zu dem in finanziellen Nöten lebenden Buben kommt zu Weihnachten durch Zufall ein reicher Onkel, der ihm bisher unerreichbare Wünsche erfüllen möchte. Als das Glück schon greifbar nahe ist, stirbt der ausgehungerte Junge plötzlich an einem Herzschlag, weil er so viel Glück nicht fassen kann. Auch die Geschichte Die alte Uhr ist in ärmlichen Verhältnissen angesiedelt. Heimlich verkauft die Mutter die geerbte Uhr des todkranken Vaters, weil er nur durch ein bestimmtes Medikament, für welches das Geld fehlt, überleben kann. Der Vater übersteht die Krankheit, ist aber wegen der Uhr sehr vergrämt. $\mathrm{Zu}$ Weihnachten überrascht ihn die Tochter mit der zurückgekauften Uhr. Sie hat heimlich gestrickt und die Sachen verkauft, um das Erbstück wieder auslösen zu können. Diese Geschichte findet sich in etwas abgewandelter Form unter dem Titel Kindesliebe in der 24 Seiten umfassenden und monatlich erscheinenden Zeitschrift Der junge Bürger wieder, die in einer Anzeige beworben wird: »Er bringt Unterhaltungsstoff, ernsten und heiteren, aber stets auf sittlicher Grundlage fußend, oder er belehrt über Wissensgebiete, die Gemeingut der Menschheit sein sollten. ${ }^{13}$ Auch

11 | Katscher, Berta: Der versteigerte Rock. Humoreske. In: Justinus, Oskar u.a.: Reisegeschichten. Wien: Verlag der Österreichisch-Ungarischen Volksbücher [1890], S. 41-52.

12 | Katscher, Berta: Weihnachtsgeschichten. Leipzig: Verlag der Zehnpfennig-Bibliothek 1890; s. auch dies.: Weihnachts-Geschichten. Wien: Verlag der Österreichisch-Ungarischen Volksbücher [0.J.].

13 | Kemmerling-Unterthuner, Ulrike: Lesen in Dornbirn. Anmerkungen zu Dornbirner Bibliotheken und Vereinsbüchereien im 19. und 20. Jahrhundert. In: Dornbirner Schriften. Beiträ- 
hier erkrankt der Vater, Herr Woltmann, ein Buchhalter, der seine Arbeit verloren hat und in ärmsten Verhältnissen lebt, schwer. Seine Frau verkauft seine Uhr, um die lebensrettenden medizinischen Tropfen zu erhalten. Nur der zehnjährige Sohn Oskar verhindert, dass das Ehepaar völlig verzweifelt. Heimlich verdient er mit dem Abschreiben wichtiger Dokumente Geld und kann die Uhr zur großen Überraschung seiner Eltern wieder auslösen. Der Vater lobt ihn mit folgenden Worten: »Aus dir wird eh' was Tüchtiges, Oskar! « ${ }^{14}$ In beiden Geschichten übernehmen also die Kinder Verantwortung für das Wohl der Familie und verzichten auf ihre eigenen Bedürfnisse. Auch die Geschichte Irma's Ostersonntage $e^{15}$ handelt von Verzicht. Ein 16-jähriges Mädchen bekommt ein Kind von einem Verehrer, der sich als Trunkenbold erweist und es schließlich verlässt. Als er eines Tages wiederkommt, will das Mädchen ihn trotz seiner Alkoholsucht und aller Warnungen der Familie heiraten, damit das Kind einen Vater hat. Die Hochzeit findet noch dazu am Tag des eigenen Geburtstages und am Sterbetag der geliebten Mutter statt. Er stirbt jedoch kurz nach der Hochzeit an den Folgen seines Alkoholkonsums, an einem Herzschlag. Das Mädchen, das sozusagen den eigenen Fehltritt mit der Hochzeit gebüßt hat, wird schließlich die Frau eines Müllers und beginnt ein neues, glückliches Leben.

Treuebruch wird, je nachdem ob sie von der Frau oder dem Mann begangen wird, unterschiedlich gewertet. Das zeigt auch die Erzählung In der Löwengrube, ${ }^{16}$ die von Paul Beyer, einem aus Wien gebürtigen Regimentsarzt in der Hercegovina, handelt. Seine Frau, eine Dalmatinerin, hilft ihm im Alltag mit Übersetzungen, da er die bosnische Sprache nicht beherrscht. Als er am Markt Baron Feldern trifft, der eben den Schleier einer schönen türkischen Frau lüften will, kommt es nur aufgrund seines Eingreifens zu keinem Eklat. Als die Frau ihn und seine Frau aus Dankbarkeit zu sich einlädt, betrügt der Ehemann seine Frau mit der Türkin. Ein Smaragd, der ihm beim Abschied überreicht wird, soll ihn in Zukunft vor Untreue schützen. Nicht der eigene Wille soll also in Zukunft einen Seitensprung verhindern, sondern die Kraft eines Edelsteins. Zugleich wird in dieser Geschichte die Vertrautheit der Autorin mit fremden Kulturen erkennbar und ihre Sensibilität für ungleiche und ungerechte Geschlechterverhältnisse. In mehreren Texten Berta Katschers ist die gesellschaftliche Tendenz erkennbar, dass Frauen >gezähmt< werden müssen. In Amor im Schnee oder: Der Schlitten als Heiratsvermittler. Eine südungarische Wintergeschichte ${ }^{17}$ ist ein Mädchen aus der Stadt zu Gast bei anderen Mädchen am Land und langweilt sich zunächst. Schließlich sitzt es während einer Schlittenfahrt scheinbar zufällig neben einem Herrn, vor dem es sich bei seinem ersten Ball durch ein Ungeschick beim Tanz blamiert hat und dem es eigentlich hat

ge zur Stadtkunde 13 (1992), S. 3-35, hier S. 14; s. auch www.bodenseebibliotheken.de/ viewer.html?page=dosc-h013-t-A014 (zuletzt eingesehen am 10.6.2015).

14 | Katscher, Berta: Kindesliebe. In: Der junge Bürger 9 (1898), S. 199-204, hier S. 204.

15 | Katscher, Berta: Irma's Ostersonntage. In: Glücksmann, Heinrich u.a.: Liebesleid. Erzählungen. Wien: Szelinski [0.J.], S. 14-28.

16 | Katscher, Berta: In der Löwengrube. Eine Haremsgeschichte. In: Folteicineano, Max u.a.: Weibergeschichten. Wien: Szelinski [0.J.], S. 32-48.

17 | Katscher, Berta: Amor im Schnee oder: Der Schlitten als Heiratsvermittler. Eine südungarische Wintergeschichte. In: dies. u.a.: Lustige Heiratsgeschichten. Wien: Szelinski [0.J.], S. 3-20. 
ausweichen wollen. Die beiden verloben sich noch während der Schlittenfahrt. Das Mädchen wird bei der Rückkehr vom Ausflug durch die Anwesenheit der gesamten Familie überrascht, denn die ganze Sache war schon lange vor dem Ausflug geplant gewesen. Ein widerspenstiges Mädchen wird so rasch wieder auf den rechten Weg geführt.

Auch in der Erzählung Die Studentin ${ }^{18}$ wird einerseits das Fehlverhalten einer jungen Frau aufgezeigt und andererseits das Thema »Blaustrumpf« erneut aufgegriffen. Leo Magnus ist zu einem Bankett geladen, man erwartet, dass er seiner Jugendfreundin Maud einen Heiratsantrag macht. Da diese jedoch einen Beruf erlernen möchte, scheut er vor einer Hochzeit zurück und schickt seinen Vetter Fred Hobson zum Bankett. Dieser ist mit Clare verlobt, die sich später als Betrügerin entpuppt. Bei einem Schiffsuntergang steht Maude dem heimlich von ihr geliebten Fred tapfer und mutig zur Seite und erobert schließlich sein Herz.

Eher anspruchslose Geschichten wurden in der Zehnpfennig-Bibliothek Nr. 6 unter dem Gesamttitel Allerlei schwierige Künste veröffentlicht. ${ }^{19}$ Unter dem Pseudonym Albert Kellner wurde darin Die Kunst, reich $z u$ werden ${ }^{20}$ publiziert - ein Plädoyer für Rechtschaffenheit und Sparsamkeit - und unter Ludwig Ungar Die Kunst, sich zu kleiden, ${ }^{21}$ in dem v.a. vor dem Tragen eines Korsetts gewarnt wird, da dies sogar zu Schlaganfällen führen kann.

Unter Ludmilla Koelle veröffentlichte Berta Katscher mit dem Titel Mein längster $\operatorname{Tag}^{22}$ die Geschichte von Laetizia, die ihren Vetter zunächst verabscheut, aber innerhalb eines Tages lieben lernt. Auch der Text Herzensschläge, ${ }^{23}$ in dem sich eine frisch verheiratete Kammerdienerin bei ihrer Arbeitgeberin bitter darüber beklagt, dass ihr Mann sie nicht zu lieben scheint, weil er sie noch nie geschlagen hat, ist eher als seicht und tragikomisch zu bezeichnen. Meine Verirrung. Eine tragikomische Geschichte ${ }^{24}$ ist eher oberflächlich gehalten. In letztgenannter Erzählung schreibt eine Frau als Überraschung für den Hochzeitstag eine Geschichte in Anlehnung an die eigene Biografie, da ihr Schreiben von ihrem Mann sehr geschätzt wird. Dieser findet jedoch den Text noch vor dem Festtag, hält ihn für einen Brief an einen heimlichen Geliebten und ist zutiefst bestürzt, bis sich alles in Wohlgefallen auflöst. In Die »Schwaben «im Banat ${ }^{25}$ erzählt sie von schwäbischen Bräuchen.

18 | Katscher, Berta: Die Studentin. Original-Erzählung. Berlin: A. Weichert [1900].

19 | Katscher, Leopold u.a.: Allerlei schwierige Künste. Wien: Oesterreichisch-Ungarisches Verlagshaus [0.J.].

20 | Kellner, Albert [Berta Katscher]: Die Kunst, reich zu werden. In: Katscher u.a.: Allerlei schwierige Künste, S. 23-37.

21 | Ungar, Ludwig [Berta Katscher]: Die Kunst, sich zu kleiden. In: Katscher u.a.: Allerlei schwierige Künste, S. 38-52.

22 Koelle, Ludmilla [Berta Katscher]: Mein längster Tag. In: Twain, Mark u.a.: Heitere Liebesgeschichten. Wien: Szelinski [0.J.], S. 21-40.

23 | Katscher, Berta: Herzensschläge. In: dies. u.a.: Aus jungen Ehen. Humoristische Erzählungen. Wien: Österreichisch-Ungarische Volksbücher 1890, S. 3-16.

24 Katscher, Berta: Meine Verirrung. Eine tragikomische Geschichte. In: dies. u.a.: Aus jungen Ehen, S. 17-35.

25 | Katscher, Berta: Die "Schwaben « im Banat. In: Kaden, Woldemar u.a.: Deutsches Leben Da und Dort. Wien: Oesterreichisch-Ungarisches Verlagshaus [0.J.], S. 53-62. 
Der verlorene $\mathrm{Hut}^{26}$ ist ebenfalls humorvoll geschrieben. Ein Mann schimpft mit seiner Frau, da sie ständig Dinge verlegt, bis er selbst einmal seinen Hut verliert.

Einen gänzlich anderen Ton schlägt sie in Zwei Muster-Arbeitgeber ${ }^{27}$ an, das sie gemeinsam mit Leopold Katscher verfasst hat. Hier wird über zwei Pioniere im Bereich arbeitnehmerfreundlicher Betriebe berichtet. Der eine, John Henry Patterson (1844-1922), Gründer der National Cash Register Company, ließ Licht und Luft in die Arbeitssäle und führte zahlreiche weitere Verbesserungen, v.a. für Arbeiterinnen, ein. Er ließ einen Speisesaal einrichten, verkürzte die Arbeitszeit, achtete streng auf Sauberkeit und Hygiene und vieles mehr. Laut Berta und Leopold Katscher setzte er sich v.a. deshalb so vehement für die Frauen ein, weil Patterson seine geliebte Frau sehr früh verloren hatte. Der andere vorgestellte Arbeitgeber ist Albans Chaix, der die Gewinnbeteiligung für Arbeitnehmerinnen und Arbeitnehmer einführte. In der Reihe Sozialer Fortschritt hatte Leopold Katscher bereits mehrere Ausgaben bestritten, u.a. Japanische Wirtschafts- und Sozialpolitik ${ }^{28}$ (8), Die so genannten Sozial-Museen ${ }^{29}$ (14), Gewinnbeteiligung ${ }^{30}$ (28/29), Abbe's »Carl ZeissStiftung in Jena $^{31}$ (34) und Erwerbs- und Wirtschaftsgenossenschaften in Deutschland und Österreich ${ }^{32}(38 / 39)$.

1907 erschien Kinderschutz und Kinderarbeit in England. Neuere kleine Beiträge von Leopold und Berta Katscher. ${ }^{33}$ Darin erzählt das Ehepaar von einigen Fällen von Verwahrlosung, Misshandlung und völliger Überarbeitung von Kindern sowie vom Nutzen der wohltätigen Vereine, die sich dieser Probleme annehmen.

Ihrer beider Hauptanliegen bestand jedoch in der Erhaltung des Friedens. In Unter der Friedenspalme. Ein Märchen für »Grosse«, das in dem von Leopold herausgegebenen Band Bertha von Suttner, die »Schwärmerin« für Güte (1903) abgedruckt ist, erzählt Berta im Märchenstil von einer Prinzessin, der neben Tugenden wie Reichtum, Schönheit oder Anmut von einer Fee auch eine »gesunde Vernunft« mitgegeben wurde. Die Vernunft sorgt dafür, dass die Prinzessin keine Freude an nichtssagenden Vergnügungen hat, sondern v.a. den Wunsch in sich spürt, den Menschen Frieden zu bringen. Um Ruhe zu finden, besucht sie häufig einen Palmenwald und denkt nach:

26 | Katscher, Berta: Der verlorene Hut. In: Wengraf, Edm[und] u.a.: Aus Bädern und Sommerfrischen. Wien: Oesterreichisch-Ungarisches Verlagshaus [0.J.], S. 57-64.

27 | Katscher, Berta/Katscher, Leopold: Zwei Muster-Arbeitgeber. Gautzsch bei Leipzig: Felix Dietrich 1908.

28 | Katscher, Leopold: Japanische Wirtschafts- und Sozialpolitik. Leipzig: Sozialer Fortschritt 1904.

29 | Katscher, Leopold: Die sogenannten "Sozial-Museen " (Museen für Arbeiterwohlfahrt und Sozialpolitik und das Pariser Musée Social als Vorbild). Leipzig: Dietrich 1904.

30 | Katscher, Leopold: Die Gewinnbeteiligung (Mit besonderer Berücksichtigung Deutschlands). Leipzig: Sozialer Fortschritt 1904.

31 | Katscher, Leopold: Abbe's "Carl Zeiss-Stiftung" in Jena. Leipzig: Sozialer Fortschritt 1905.

32 Katscher, Leopold: Wirtschaftsgenossenschaften in Deutschland und Österreich. Leipzig: Sozialer Fortschritt 1905.

33 | Katscher, Leopold/Katscher, Berta: Kinderschutz und Kinderarbeit in England. Neuere kleine Beiträge. Prag: Verlag des Vereines 1907. 
Ja, den Frieden, das war's, was die Welt brauchte. Die kleinen und grossen Kämpfe verrohten die Menschen, pflanzten Selbstsucht und Ehrgeiz in ihre Herzen. Wenn erst Friede, ewiger Friede herrschte, dann liesse sich's herrlich leben! Die gesunde Vernunft zeigte der Prinzessin, was der Welt not tat, aber was nützte das, wenn nicht alle Menschen es wussten! ${ }^{34}$

Eines Tages hat sie einen Traum: Mutter Gaea, die Erde, weint, und die Sonne möchte ihre Tränen trocknen, da erzählt Gaea der Sonne von den Kriegen auf der Erde und wie unglücklich sie darüber sei. Die Sonne ist empört über die Schlechtigkeit der Menschen und würde sich am liebsten von der Erde abwenden, aber damit wäre auch die Natur selbst verloren. Gemeinsam überlegen die Erde und die Sonne, wie sie Frieden in die Welt bringen können, dabei kommt der Sonne der Gedanke, die Prinzessin um Hilfe zu bitten. Gaea wendet ein: »Ich glaube nicht, dass ihr das gelingen wird. [...] Bedenke doch, sie ist nur ein schwaches Weib.« ${ }^{35}$ Darauf erwidert die Sonne Bertha von Suttner zitierend:

Ei wirklich? Nur ein schwaches Weib?! So schlecht kennst du deine Menschen, dass du nicht weisst, wie stark gerade ein Weib sein kann, wenn es ein bestimmtes Ziel vor Augen hat, das es erreichen will? Ich sage dir, nur ein Weib vermag uns von dem Gespenst zu befreien! Nur ein Weib ist imstande, durch zähes Festhalten an den einmal gefassten Ideen Feinde aus dem Felde zu schlagen und all den Hohn und Spott auf sich zu nehmen, den die verblendeten Menschen anfangs auf sie schleudern werden. Das mutige Beispiel dieses Weibes wird auch ihre Mitschwestern anspornen, für eine Sache zu kämpfen, zu deren Trägerinnen sie in erster Reihe berufen sind. Wenn Frauen die Friedensfahne erheben und Rekruten für sie werben, ist die Sache so gut wie gewonnen! ${ }^{36}$

Die Prinzessin erwacht und erkennt schließlich: »Ich werde nimmer ruhen und rasten können, bis $-<$ da raschelte etwas in ihrem Baum und ein frisches Palmenblatt fiel ihr in den Schoss - >ja bis ich die Friedenspalme zum Lieblingsbaum der ganzen Welt gemacht haben werde! « ${ }^{37}$

Das Streben nach Frieden war tief in ihr verankert. 1895 veröffentlichte sie in der Zeitschrift Die Waffen nieder! die Rezension Lubbock und unsere Sache über John Lubbock, Naturforscher, Kulturhistoriker und Bankier und sein Buch The Use of Life, erstmals 1894 in London bei Macmillan erschienen und mehrmals neu aufgelegt.

Mit der in Prag in eine adelige, wohlhabende Familie geborenen Bertha von Suttner (1843-1914) verband Berta Katscher einiges. Auch Bertha von Suttner stammte aus schwierigen familiären Verhältnissen. Der große Alters- und Standesunterschied ihrer Eltern führte dazu, dass ihre Mutter nie wirklich in die Familie aufgenommen wurde. Auch ihr Ehemann Arthur Gundaccar von Suttner (1850-1902), den sie 1876 heiratete und der selbst journalistische Texte und Abenteuerromane verfasste, unterstützte die schriftstellerischen und gesellschaftspolitischen Bestre-

34 | Katscher, Berta: Unter der Friedenspalme. Ein Märchen für "Grosse ". In: Katscher, Leopold: Bertha von Suttner, die "Schwärmerin« für Güte. Dresden: Pierson 1903, S. 119-126, hier S. 121.

35 | Ebd., S. 125.

$\mathbf{3 6} \mid$ Ebd.

37 | Ebd., S. 126. 
bungen seiner Frau. Auch sie schrieb für zahlreiche Zeitungen und Zeitschriften. In ihren Schriften widmete sie sich hauptsächlich den sozialen Missständen und dem Pazifismus. 1891 gründete sie die Österreichische Friedensgesellschaft, deren Präsidentin sie zeitlebens blieb. Von 1892 bis 1899 gab sie die Zeitschrift Die Waffen nieder! (später Friedens-Warte) heraus, für die sie Glossen, Aufsätze und Kommentare zum politischen Zeitgeschehen lieferte. Bertha von Suttner war Rednerin und Teilnehmerin an allen Weltfriedenskongressen, in internationalen Diskussionsforen und interparlamentarischen Konferenzen. Nachdem ihr Mann 1902 gestorben war, zog sie nach Wien, veröffentlichte u.a. in den deutschsprachigen Zeitungen Ungarns. Die enge Zusammenarbeit mit dem Ehepaar Katscher zeigte sich auch in gemeinsamen Publikationen, so gab Leopold Katscher 1896 etwa ihre Erzählungen, Aphorismen und Betrachtungen unter dem Titel Krieg und Frieden heraus. ${ }^{38}$ In ihren Romanen setzt sie sich kritisch mit Problemen wie Nationalismus, Antisemitismus, Frauenemanzipation und Jugenderziehung auseinander. Ihr Roman Die Waffen nieder! erreichte bis 189614 Auflagen, wurde in fast alle Sprachen Europas übersetzt und trug wesentlich zur Verbreitung und Popularisierung der Friedensidee und -bewegung in Europa und Amerika bei. Auch sie setzte sich wie Berta Katscher dafür ein, die pazifistischen Gedanken der Jugend näherzubringen und schrieb u.a. das Vorwort zu dem Jugendbuch Pacifistisches Jugendbuch. Ein Ratgeber für Eltern und Erzieher (1910) von Arthur Müller. ${ }^{39}$ Ihr Jugendbuch Marthas Kinder. Eine Fortsetzung zu »Die Waffen nieder!« erschien erstmals 1903 in Dresden und wurde mehrmals neu aufgelegt. ${ }^{40}$

\section{Kein Soldatenspiel - eine Warnung an die Jugend}

In Soldatenkinder. Eine Erzählung für die reifere Jugend $(1897)^{41}$ verurteilt Berta Katscher den Krieg auf das Schärfste. Die Erzählung beginnt mit einem Unfall beim Soldatenspielen mehrerer Buben. Einer von ihnen bricht sich das Bein, die Mutter, die sehr erschrocken ist, als ihr Sohn auf einer Bahre liegend heimgebracht wird, erzählt ihm und seinen Freunden eine Geschichte, die in ihrer Familie passiert ist. Ihr Ehemann und ihr Bruder mussten bei der Schlacht von Solferino gegeneinander kämpfen, dabei wurde ihr Bruder getötet. Ihr Ehemann hat das Drama nie überwunden und verfiel in Depressionen. Damit greift sie ein Motiv aus Die Waffen nieder! auf.

Oskar, einer der Knaben, wird wirklich Soldat, muss aber bald einsehen, dass das Exerzieren ihm nicht liegt. Da erzählt ihm ein Kamerad das Märchen von der Kanonenkugel und der Nähnadel. Die Nadel, die früher zum Nähen fester Stoffe

38 | Suttner, Bertha Freifrau von: Krieg und Frieden. Erzählungen, Aphorismen und Betrachtungen. Hg. v. Leopold Katscher. Berlin: Rosenbaum 1896.

39 | Suttner, Bertha von: Vorwort. In: Müller, Arthur: Pacifistisches Jugendbuch. Ein Ratgeber für Eltern und Erzieher. Wien: Hassel 1910, S. III-V.

40 | Suttner, Bertha von: Martha's Kinder. Eine Fortsetzung zu „Die Waffen nieder!«. Dresden: Pierson 1903. Vgl. Handbuch der österreichischen Kinder- und Jugendbuchautorinnen. Bd. 2: M-Z, S. 1124-1129.

41 | Katscher, Bertha: Soldatenkinder. Eine Erzählung für die reifere Jugend. Stuttgart: Süddeutsches Verlags-Institut 1897. 
verwendet wurde, wollte es besser haben und versteckte sich in einer Hosenstulpe. Sie lernte zwar ein Stück von der Welt kennen, wurde aber wegen ihres Hochmutes verspottet. Im Gespräch mit der Kanonenkugel wird ihr bewusst, dass sie nur gemeinsam mit vielen anderen den Menschen dienlich sein kann, da sie im Gegensatz zur Kanonenkugel kein Leid bringt, sondern ein sehr nützlicher Gegenstand ist. Die Geschichte endet damit, dass die inzwischen auf die Erde gefallene Nadel darauf hofft, gefunden zu werden, bevor sie verrostet ist. In der Rahmengeschichte wird weitererzählt, dass dem Vater von Oskar und seiner Schwester Doris in der Schlacht bei Königgrätz eine Kugel ins Bein gedrungen ist. Eines Tages bricht die Wunde wieder auf, die Kugel, die nicht entfernt werden konnte, verursacht eine Infektion und der Vater stirbt qualvoll. Daraufhin will Doris ihren Bruder daran hindern, Soldat zu werden, er wird jedoch eingezogen und hält seine Erlebnisse in Form eines Tagebuches fest. Fritz, ein Jugendfreund der beiden, ist inzwischen Chirurg und heimlich in Doris verliebt. Auch er wird zum Militärdienst eingezogen, Doris folgt ihm als freiwillige Pflegerin. Oskar wird von einer Türkin, deren Mann eben ermordet wird, mit einem Dolch schwer verletzt. Fritz kann für seinen Freund Oskar nichts mehr tun. Kurz vor seinem Tod verlobt sich Oskar jedoch zur letzten großen Freude seines Freundes mit Doris. Sie trifft schließlich noch auf die Türkin, die Oskar verwundet hat und diese Tat bitter bereut. Oskar kann ihr am Sterbebett noch verzeihen. Er wünscht sich folgenden Text für seinen Grabstein: »Hier ruht ein Soldatenkind, das in blindem Ungestüm den rechten Weg verfehlt hat und als es ihn wieder gefunden, war's zu spät. Frieden, Frieden, sei der Menschheit Losungswort. ${ }^{42}$ Auf der Homepage der »Ausgewählten Erwerbungen der Staatsbibliothek zu Berlin - Preußischer Kulturbesitz« kann man lesen: »Obwohl der Titel >Soldatenkinder < darauf hindeutet, dass es sich auch bei diesem Band um eine patriotische Jugenderzählung handelt, gehört Berta Katschers Buch zu den wenigen pazifistischen Publikationen dieser Zeit. « ${ }^{43}$

1897 veröffentlichte Berta Katscher in der Augustausgabe der Jugendzeitschrift Der junge Bürger, die von der Zeitschrift Vorarlberger Volksfreund als »anerkannt vortreffliche Jugendschrift « ${ }^{44}$ bezeichnet wurde, eine weitere pazifistische Erzählung, Bei Solferino, in der sie die Niederlage der Österreicher bei der Schlacht von 1859 schilderte. ${ }^{45}$

42 | Ebd., S. 111.

43 | NN: Ausgewählte Erwerbungen der Staatsbibliothek zu Berlin Preußischer Kulturbesitz, www.ag-sdd.de/Subsites/agsdd/DE/Erwerbung/Berlin/erwerbungBerlin.html?cms_not First=true\&cms_docld=200396 (zuletzt eingesehen am 4.5.2016).

44 | NN: Der junge Bürger. In: Vorarlberger Volksfreund v. 6.8.1897, S. 7.

45 | Der junge Bürger. Hg. vom Lehrerverein des Landes Vorarlberg. Dornbirn: Daniel Feuerstein 1897. 


\section{Reiseabenteuer - Ermutigungen für die Jugend}

1892 erschien Hermann Vambergs Leben und Reiseabenteuer. Der Jugend erzählt. ${ }^{46}$ Berta Katscher berichtete darin vom Leben und Wirken des Forschungsreisenden, Orientalisten, Turkologen, Linguisten, Ethnologen, Geografen, Schriftsteller, Hochschullehrer Ármin Vámbéry (1832-1913), der auch unter Hermann Vamberg(er) beziehungsweise Bamberger bekannt wurde. Obwohl unter ärmlichen Bedingungen und mit zahlreichen Geschwistern aufgewachsen, bereits mit zwölf Jahren Lehrling bei einem Schneider und seit Kindheit gehbehindert, scheute er keine Hindernisse und strebte stets nach Bildung. Zunächst war er als Hauslehrer tätig, besuchte das Gymnasium, sprach fließend Latein und kam mit 14 Jahren nach Preßburg/Bratislava. In Konstantinopel war er schließlich als Lehrer tätig und um 1860 der einzige Europäer, der sich frei in der türkischen Gesellschaft bewegen konnte und dem es erlaubt war, Bibliotheken und Archive zu benutzen. Ihm wurde der ehrende Titel »Efendi« verliehen, er reiste weiter nach Persien und erlebte mehrere gefährliche Abenteuer. Schließlich wurde er zu einem Experten für den asiatischen Raum.

Vamberg entstammte einer armen orthodoxen jüdischen Familie, er erlernte neben zahlreichen europäischen Sprachen auch Arabisch, Türkisch und Persisch, die er fließend beherrschte. Er verfasste z.B. Übersetzungen von Werken über die türkische Geschichte und veröffentlichte ein deutsch-türkisches Wörterbuch. Von seinen Reisen in ferne Länder brachte er geografisches, ethnografisches und linguistisches Wissen mit. Sein Reisebericht Travels and Adventures in Central Asia ${ }^{47}$ wurde in ganz Europa mit großem Interesse aufgenommen. Nach seiner Rückkehr aus Asien im Frühling 1864 erhielt er eine Professur an der Universität Budapest. Von 1865 bis 1905 war er in Budapest Professor für orientalische Sprachen. ${ }^{48}$

Auch Berta Katschers kurze Kindergeschichte mit dem Titel Guck-in-die-Welt ${ }^{49}$ (1912), die in der Zeitschrift Kinderland postum erschien und an Heinrich Hoffmanns Die Geschichte Hans-Guck-in-die-Luft in Struwwelpeter erinnert, berichtet von einer lohnenden Reise. Jancsi (Hans) wächst in einer einfachen Bauernfamilie mit zahlreichen Geschwistern im Flachland Ungarns auf. Als Einziger ist er träumerisch und körperlich schwach. Auf einer erbettelten Reise mit seinem Vater zu seiner Tante sieht er zum ersten Mal einen Berg und beschließt, ihn in der Nacht alleine $\mathrm{zu}$ besteigen, da ihm diese Wanderung niemand zutraut. Ermattet wird er von einem slavisch sprechenden Schafhirten gefunden und in seine Hütte gebracht. Die ungarische Magd bringt ihn schließlich zu Vater und Tante zurück. Anstatt zu schimpfen, wie der Knabe befürchtet hat, sind die beiden nur glücklich, ihn unversehrt wieder zu haben. Die Geschichte endet mit den Worten: »Der nächtli-

46 | Katscher, Berta: Hermann Vamberg's Leben und Reiseabenteuer. Der Jugend erzählt. Teschen/Wien: Prochaska 1892.

47 | Vámbéry, Arminius: Travels in Central Asia being the Account of a Journey from Teheran ... London: John Murray 1864.

48 | Vgl. Bartholomä, Ruth: Von Zentralasien nach Windsor Castle. Leben und Werk des ungarischen Orientalisten Arminius Vámbéry (1832-1913). Würzburg: Ergon 2006.

49 | Katscher, Berta: Guck-in-die-Welt. In: Kinderland. Blätter für ethische Jugenderziehung. Monatsbeilage zu "Ethische Kultur " v. 1.1912, S. 39-40; s. auch http://goobiweb.bbf.dipf.de/ viewer/image/ZDB025299433_0012/39/LOG_0063/ (zuletzt eingesehen am 4.5.2016). 
che Ausflug war Jancsis erstes, aber nicht sein letztes Abenteuer. Unser Guck in die Welt, wie ihn seine Tante nannte, ist heute ein weltbekannter Künstler. Die Bilder, die er träumend in der weiten Puszta erschaut, zieren die Wände mancher Kunstausstellung und ein Träumer ist er geblieben. ${ }^{50}$

In der Geschichte Die Lebensretterin ${ }^{51}$ ist das Stadtkind Elschen zu Besuch bei seinem strengen Onkel am Bauernhof und verübt dort zahlreiche Streiche. Als es eines Tages nicht zum Essen heimkommt und sich die Familie schon sehr sorgt, findet man das Mädchen schlafend auf dem Eierkorb im Hühnerstall sitzend. Es wollte nur die kleinen »Küchlein« in den Eiern wärmen, so die Erklärung.

\section{FAzIT}

Berta Katscher ist heute trotz ihres breiten Schaffens und ihrer ehemaligen Bedeutung als eine der Pionierinnen im Bereich der Friedenserziehung, als Verfasserin zahlreicher Beiträge in Zeitungen und Zeitschriften und auch als Autorin von Kinder- und Jugendliteratur nahezu unbekannt. Es existieren kaum wissenschaftliche Abhandlungen über die vielseitige und produktive Schriftstellerin.

Berta Katscher war mit ihren auf dem ersten Blick etwas oberflächlich erscheinenden Beiträgen in vielen Fachgebieten sehr fortschrittlich. Ihr gelang es, das zeitgenössische gesellschaftliche Leben kritisch zu analysieren und zum Teil auf humoristische Weise darzustellen. Interessant ist auch, dass das vielseitige Ehepaar Katscher schon sehr früh für den Schutz von geistigem Eigentum, den Urheberschutz und für die finanzielle Absicherung von Schriftstellerinnen und Schriftstellern sowie Künstlerinnen und Künstlern in Form von Lizenzabgaben und Pensionskassen eintrat. Auch gegen die Spiel- und Trunksucht sowie gegen Kindermisshandlung und Gewalt gegen Tiere trat Berta Katscher vehement ein.

Der 43 Boxen umfassende Nachlass enthält v.a. Briefwechsel, Manuskripte, Artikel und finanzielle und juristische Unterlagen von Leopold Katscher, aber auch Berta Katschers Nachlass ist hier zu finden. Ursprünglich als Teil der SchwimmerLloyd-Collection an die New York Public Library gekommen, wurde er 2006 als separate Sammlung aufgenommen. Im Nachlass sind auch die Schreibweisen Kacser, Kácser, Kácer und Kácsa zu finden. Das Werk von Leopold und Berta Katscher ist oft schwer zu trennen, da sich die beiden die Pseudonyme teilten - wodurch aber auch eine tiefe Übereinstimmung zwischen ihren Gedanken und eine sehr enge Zusammenarbeit deutlich wird.

Die langjährige Korrespondenz mit Bertha von Suttner ist ebenso enthalten wie private Briefe oder Verlagskorrespondenz. Der umfangreiche Briefwechsel mit zeitgenössischen Vertreterinnen und Vertretern von Kunst und Kultur sowie die ausgedehnte Verlagskorrespondenz wären für weitere Forschungen durchaus interessant. Darüber hinaus scheint es lohnenswert zu fragen, inwieweit Berta Katscher mit anderen Schriftstellerkolleginnen und -kollegen in Kontakt war. Man

$\mathbf{5 0}$ | Ebd., S. 40.

51 Katscher, Berta: Die Lebensretterin. In: Kinderland. Blätter für ethische Jugenderziehung. Monatsbeilage zu "Ethische Kultur" v. 1.1913, S. 31-32; s. auch https://www. digizeitschriften.de/dms/img/?PID=ZDB025299433_0013|LOG_0005\&physid=PH YS_0001\#navi (zuletzt eingesehen am 4.5.2016). 
denke hier z.B. an Hedwig Pötting (1853-1915), die Freundin und Unterstützerin von Bertha von Suttner, Vorstandsmitglied der Friedenskommission im Bund der Österreichischen Frauenvereine und Autorin des ersten pazifistischen Jugendbuchs im deutschen Sprachraum Marthas Tagebuch, einer Bearbeitung ihres Buches Die Waffen nieder!. Insgesamt ist zu hoffen, dass Berta Katschers Leben und Werk in zukünftigen wissenschaftlichen Arbeiten die ihr gebührende Berücksichtigung findet.

\section{LITERATUR}

[Annonce]. In: Pester Lloyd v. 2.5.1897, S. 8.

[Annonce]. In: Salzburger Volksblatt 3.12.1886, [S. 5].

Bartholomä, Ruth: Von Zentralasien nach Windsor Castle. Leben und Werk des ungarischen Orientalisten Arminius Vámbéry (1832-1913). Würzburg: Ergon 2006.

Blumesberger, Susanne: Handbuch der österreichischen Kinder- und Jugendbuchautorinnen. 2 Bde. Wien: Böhlau 2014.

Der junge Bürger. Hg. vom Lehrerverein des Landes Vorarlberg. Dornbirn: Daniel Feuerstein 1897.

Hacker, Lucia: Schreibende Frauen um 1900. Rollen - Bilder - Gesten. Berlin u.a.: LIT 2007.

Kácser, Berta: A hajtóvadászat [Die Treibjagd]. In: Az Ezeréves Magyarország 2 (1897), S. 21.

Katscher, Berta: Amor im Schnee oder: Der Schlitten als Heiratsvermittler. Eine südungarische Wintergeschichte. In: dies. u.a.: Lustige Heiratsgeschichten. Wien: Szelinski [o.J.], S. 3-20.

Katscher, Berta: Der verlorene Hut. In: Wengraf, Edm[und] u.a.: Aus Bädern und Sommerfrischen. Wien: Oesterreichisch-Ungarisches Verlagshaus [0.J.], S. 5764 .

Katscher, Berta: Die »Schwaben« im Banat. In: Kaden, Woldemar u.a.: Deutsches Leben Da und Dort. Wien: Oesterreichisch-Ungarisches Verlagshaus [o.J.], S. 53-62.

Katscher, Berta: Herzensschläge. In: dies. u.a.: Aus jungen Ehen, S. 3-16.

Katscher, Berta: Meine Verirrung. Eine tragikomische Geschichte. In: dies. u.a.: Aus jungen Ehen, S. 17-35.

Katscher, Berta: In der Löwengrube. Eine Haremsgeschichte. In: Folteicineano, Max u.a.: Weibergeschichten. Wien: Szelinski [o.J.], S. 32-48.

Katscher, Berta: Irma's Ostersonntage. In: Glücksmann, Heinrich u.a.: Liebesleid. Erzählungen. Wien: Szelinski [o.J.], S. 14-28.

Katscher, Berta: Klagebrief einer Strohwitwe. In: Prager Tagblatt v. 24.7.1886, S. 1-2.

Katscher, Berta: Der versteigerte Rock. Humoreske. In: Justinus, Oskar u.a.: Reisegeschichten. Wien: Verlag der Österreichisch-Ungarischen Volksbücher [1890], S. 41-52.

Katscher, Berta: Weihnachtsgeschichten. Leipzig: Verlag der Zehnpfennig-Bibliothek 1890. 
Katscher, Berta: Die beiden Todten. Bluette in einem Aufzug. In: Wiener Liebesgaben. Zum Besten der Wiener freiwilligen Rettungs-Gesellschaft. Wien/Leipzig: Max Merlin 1892, S. 97-114.

Katscher, Berta: Hermann Vamberg's Leben und Reiseabenteuer. Der Jugend erzählt. Teschen/Wien: Prochaska 1892.

Katscher, Berta: Kindesliebe. In: Der junge Bürger 9 (1898), S. 199-204.

Katscher, Berta: Die Studentin. Original-Erzählung. Berlin: A. Weichert [1900].

Katscher, Berta: Unter der Friedenspalme. Ein Märchen für »Grosse«. In: Katscher, Leopold: Bertha von Suttner, die »Schwärmerin« für Güte. Dresden: Pierson 1903 , S. 119-126.

Katscher, Berta: Guck-in-die-Welt. In: Kinderland. Blätter für ethische Jugenderziehung. Monatsbeilage $\mathrm{zu} »$ Ethische Kultur« v. 1.1912, S. 39-40; s. auch http:// goobiweb.bbf.dipf.de/viewer/image/ZDBo25299433_0012/39/LOG_0o6.

Katscher, Berta: Die Lebensretterin. In: Kinderland. Blätter für ethische Jugenderziehung. Monatsbeilage zu »Ethische Kultur« v. 1.1913, S. 31-32; s. auch https:// www.digizeitschriften.de/dms/img/?PID=ZDBo25299433_0013|LOG_0005\& physid=PHYS_ooo1\#navi.

Katscher, Berta/Katscher, Leopold: Zwei Muster-Arbeitgeber. Gautzsch bei Leipzig: Felix Dietrich 1908.

Katscher, Berta u.a.: Aus jungen Ehen. Humoristische Erzählungen. Wien: Oesterreichisch-Ungarisches Verlagshaus 1890.

Katscher, Bertha: Verheirathet, aber dennoch glücklich! In: Deutsche Wochenschrift. Organ für die gemeinsamen nationalen Interessen Österreichs und Deutschlands v. 28.11.1886, S. 611-612.

Katscher, Bertha: Soldatenkinder. Eine Erzählung für die reifere Jugend. Stuttgart: Süddeutsches Verlags-Institut 1897.

Katscher, Leopold: Schuldlos verurteilt! Anregungen, Betrachtungen, Erzählungen. Leipzig: Janssen 1895 .

Katscher, Leopold: Die Gewinnbeteiligung. (Mit besonderer Berücksichtigung Deutschlands). Leipzig: Sozialer Fortschritt 1904.

Katscher, Leopold: Die sogenannten »Sozial-Museen« (Museen für Arbeiterwohlfahrt und Sozialpolitik und das Pariser Musée Social als Vorbild). Leipzig: Dietrich 1904 .

Katscher, Leopold: Japanische Wirtschafts- und Sozialpolitik. Leipzig: Sozialer Fortschritt 1904.

Katscher, Leopold: Abbe's »Carl Zeiss-Stiftung« in Jena Leipzig: Sozialer Fortschritt 1905 .

Katscher, Leopold: Wirtschaftsgenossenschaften in Deutschland und Österreich. Leipzig: Sozialer Fortschritt 1905.

Katscher, Leopold/Katscher, Berta: Kinderschutz und Kinderarbeit in England. Neuere kleine Beiträge. Prag: Verlag des Vereines 1907.

Katscher, Leopold u.a.: Allerlei schwierige Künste. Wien: Oesterreichisch-Ungarisches Verlagshaus [o.J.].

Kellner, Albert [Berta Katscher]: Die Kunst, reich zu werden. In: Katscher u.a.: Allerlei schwierige Künste, S. 23-37.

Kemmerling-Unterthuner, Ulrike: Lesen in Dornbirn. Anmerkungen zu Dornbirner Bibliotheken und Vereinsbüchereien im 19. und 20. Jahrhundert. In: 
Dornbirner Schriften. Beiträge zur Stadtkunde 13 (1992), S. 3-35; s. auch www. bodenseebibliotheken.de/viewer.html? page=dosc-ho13-t-Ao14.

Koelle, Ludmilla [Berta Katscher]: Mein längster Tag. In: Twain, Mark u.a.: Heitere Liebesgeschichten. Wien: Szelinski [0.J.], S. 21-40.

Leopold and Berta Katscher Papers 1866-1939. MssCol 6318.

Meier, Jörg: Deutschsprachige Schriftstellerinnen des 18.-20. Jahrhunderts aus dem Gebiet der heutigen Slowakei. In: Hörner, Petra (Hg.): Vergessene Literatur - Ungenannte Themen deutscher Schriftstellerinnen. Frankfurt a.M. u.a.: Peter Lang 2001, S. 241-262.

NN: Ausgewählte Erwerbungen der Staatsbibliothek zu Berlin Preußischer Kulturbesitz, www.ag-sdd.de/Subsites/agsdd/DE/Erwerbung/Berlin/erwerbungBer lin.html?cms_notFirst=true\&cms_docId=200396.

NN: Die Österreichische Gesellschaft der Friedensfreunde. In: Neue Freie Presse v. $13 \cdot 4.1895$, S. 6 .

NN: Der junge Bürger. In: Vorarlberger Volksfreund v. 6.8.1897, S. 7 .

Suttner, A[rthur] G[undaccar] v.: Schuldlos verurteilt. In: Wiener Montagspost v. 12.11.1894, S. 5 .

Suttner, Bertha Freifrau von: Krieg und Frieden. Erzählungen, Aphorismen und Betrachtungen. Hg. v. Leopold Katscher. Berlin: Rosenbaum 1896.

Suttner, Bertha von: Martha's Kinder. Eine Fortsetzung zu »Die Waffen nieder«. Dresden: Pierson 1903.

Suttner, Bertha von: Vorwort. In: Müller, Arthur: Pacifistisches Jugendbuch. Ein Ratgeber für Eltern und Erzieher. Wien: Hassel 1910, S. III-V.

Ungar, Ludwig [Berta Katscher]: Die Kunst, sich zu kleiden. In: Katscher, Leopold u.a.: Allerlei schwierige Künste, S. 38-52.

Vámbéry, Arminius: Travels in Central Asia being the Account of a Journey from Teheran ... London: John Murray 1864. 